\title{
miR-425-5p promotes cell proliferation, migration and invasion by directly targeting FOXD3 in hepatocellular carcinoma cells
}

\author{
HEWEN WU, JIA SHANG, WEILI ZHAN, JUNPING LIU, HUIBIN NING and NING CHEN \\ Department of Infectious Disease, Henan Key Laboratory for Liver Disease, Henan Provincial People's Hospital, \\ People's Hospital of Zhengzhou University, Zhengzhou, Henan 450003, P.R. China
}

Received September 9, 2018; Accepted April 25, 2019

DOI: $10.3892 / \mathrm{mmr} .2019 .10427$

\begin{abstract}
MicroRNAs (miRs) are important regulators of the tumorigenesis and metastasis of various cancers. In the present study, the roles and underlying mechanisms of miR-425-5p in the development of hepatocellular carcinoma (HCC) were investigated. RT-qPCR analysis revealed that miR-425-5p was upregulated in HCC tissues and cell lines. A functional study in vitro using MTT assays, colony formation and Transwell assays demonstrated that overexpression of miR-425-5p promoted the proliferation, migration, and invasion of HCC cells, prevented cell apoptosis and accelerates the epithelial-mesenchymal transition process, whereas miR-425-5p knockdown induced opposing effects. A further mechanistic study revealed that forkhead box D3 (FOXD3) was a direct target of miR-425-5p, and gain- and loss-of-function of FOXD3 studies demonstrated that FOXD3 suppressed HCC cell proliferation, migration, and invasion. Furthermore, rescue experiments revealed that overexpression of FOXD3 counteracted the positive effects of miR-425-5p on HCC malignant behaviors. Collectively, the present results demonstrated that miR-425-5p promoted HCC cell proliferation, migration, and invasion by suppressing FOXD3 expression, potentially providing a novel target for the treatment of $\mathrm{HCC}$.
\end{abstract}

\section{Introduction}

Hepatocellular carcinoma (HCC) is one of the most common human cancers globally and the second leading cause of cancer-associated mortality (1). At present, the major treatment

Correspondence to: Dr Hewen Wu, Department of Infectious Disease, Henan Key Laboratory for Liver Disease, Henan Provincial People's Hospital, People's Hospital of Zhengzhou University, 7 Weiwu Road, Zhengzhou, Henan 450003, P.R. China

E-mail: hewenwu1973@126.com

Abbreviations: miRNA/miR, microRNA; FOXD3, forkhead box D3; $\mathrm{HCC}$, hepatocellular carcinoma

Key words: HCC, miR-425-5p, FOXD3, HCC cell malignant behaviors, cell apoptosis strategies for HCC are early detection, surgical resection, liver implantation and gene therapy (1). Despite major advances in the diagnosis and treatment of HCC, the 5-year survival of patients with HCC remains poor due to the high incidence of cancer recurrence and metastasis (1). Therefore, it is urgent to investigate the molecular mechanisms underlying HCC development. A number of reports have indicated that microRNAs (miRNAs/miRs) serve important roles in HCC carcinogenesis (2-16).

miRNAs are 22 -nucleotide, small, noncoding RNAs that can post-transcriptionally regulate target gene expression via complementary base pairing to block the translation or promote the degradation of target mRNAs (17). miRNAs have been implicated in various physiological and pathological processes, including cell differentiation, embryonic development and carcinogenesis (18). Increasing evidence demonstrates that dysregulated miRNAs are involved in the development and progression of a variety of cancers, including HCC (2). For example, miR-199-3p, an miRNA reported to be downregulated in HCC, can inhibit tumorigenesis of HCC cells by targeting zinc fingers and homeoboxes protein 1/p53-upregulated modulator of apoptosis signaling, vascular endothelial growth factor (VEGF)A, VEGF receptor (VEGFR)1, VEGFR2, hepatocyte growth factor, matrix metalloproteinase-2, yes-associated protein 1 and CD151, and increasing the doxorubicin sensitivity of human HCC cells by regulating mTOR and c-Met (3-9). Furthermore, miR-199-3p was demonstrated to efficiently inhibit tumor growth in a transgenic mouse model of HCC and an HCC tumor-bearing nude mouse model $(10,11)$. A recent report revealed that miR-501-3p was downregulated in metastatic HCC cell lines and tissue samples from patients with recurring and metastasized $\mathrm{HCC}$, and its expression was associated with tumor progression and poor prognosis in patients with HCC; further mechanistic study revealed that Lin-7 homolog A mediated the suppressive effects of miR-501-3p on the metastasis and progression of HCCs (12). Recent studies also revealed that HCC cell-derived exosomal miRNAs serve important roles in HCC development, particularly in metastasis (13-16). For example, tumor-derived exosomal miR-25-5p promoted tumor self-seeding by enhancing the invasive and migratory abilities of recipient HCC cells (14). Hepatoma cell-secreted miR-103 increased vascular permeability and promoted metastasis by directly inhibiting the expression of 
VE-cadherin, p120-catenin and zonula occludens 1 (15). It has been reported that miR-425-5p expression was dysregulated in various cancers, including gastric cancer, $\mathrm{HCC}$, cervical cancer, esophageal squamous cell carcinoma, nasopharyngeal carcinoma, renal cell carcinoma and melanoma, and that the altered expression of miR-425-5p is associated with the pathogenesis and progression of cancer (19-26); however, the roles and mechanisms of miR-425-5p in the development of HCC are yet to be determined.

Forkhead box D3 (FOXD3) is a member of the forkhead box family of transcription factors. The forkhead box proteins are characterized by a distinct conserved 'forkhead' or 'winged helix' DNA-binding domain (27). FOXD3 is an important stem cell factor expressed in various types of stem cells and has an established role in development (28). Various studies have reported that FOXD3 is also involved in the development of a number of cancers (29-37); however, reports into its roles and mechanisms in HCC are limited.

In the present study, the potential roles of miR-425-5p in the development of HCC were investigated. The present findings demonstrated that miR-425-5p was significantly upregulated in HCC tissues and cells. miR-425-5p promoted the proliferation, migration and invasion of hepatoma cells by directly targeting and repressing FOXD3, the expression of which may suppress the malignant behavior of HCC cells. The present findings may be useful in understanding the molecular mechanisms underlying the development of HCC identifying novel treatment strategies for HCC.

\section{Materials and methods}

Clinical tissues. A total of 30 pairs of HCC tissue specimens were collected from patients who had undergone complete surgical resection at Henan Provincial People's Hospital. Among the 30 pairs of HCC tissue specimens, 20 were hepatitis B virus-positive. Patient were divided into 'miR-425-5p high' and 'miR-425-5p low' groups using a threshold of 0.5 relative expression of miR-425-5p/U6. All tissue specimens (HCC tissues and adjacent nontumor tissues) were immediately snap-frozen in liquid nitrogen and stored at $-80^{\circ} \mathrm{C}$ following surgery. Written informed consent was obtained from each patient. The study was approved by the Ethics Committee of Henan Provincial People's Hospital. The clinical information are presented in Tables I and SI.

Cell culture and transfection. The human hepatoma cell lines HCCLM3, Hep3B and Huh7 cells were purchased from the American Type Culture Collection. The liver cancer cell line HepG2 and liver cell line L02 were purchased from the Chinese Academy of Sciences Cell Bank. All cells were cultured in DMEM (Invitrogen; Thermo Fisher Scientific, Inc.) containing 10\% FBS (Gibco; Thermo Fisher Scientific, Inc.) and incubated in a humidified $37^{\circ} \mathrm{C}$ incubator with $5 \% \mathrm{CO}_{2}$. Cell transfection was performed using Lipofectamine ${ }^{\circledR} 2000$ (Invitrogen; Thermo Fisher Scientific, Inc.) according to the manufacturer's protocols.

Plasmid construction. The potential targets of miR-425-5p were predicted using TargetScanHaman 7.2 (www.targetscan. org) and PicTar databases (pictar.mdc-berlin.de) $(38,39)$.
The FOXD3 gene was amplified from cDNA derived from HCCLM3 cells and cloned into pcDNA3.0 (Invitrogen; Thermo Fisher Scientific, Inc.) Pfu DNA polymerase (Promega Corporation) was used to amplify cDNA, and the PCR conditions were as follows: $95^{\circ} \mathrm{C}$ for $5 \mathrm{~min}$, followed by 30 cycles of $94^{\circ} \mathrm{C}$ for $30 \mathrm{sec}, 58^{\circ} \mathrm{C}$ for $2 \mathrm{~min}$ and $72^{\circ} \mathrm{C}$ for $30 \mathrm{sec}$, with a final step of $72^{\circ} \mathrm{C}$ for $10 \mathrm{~min}$. FOXD3-specific and negative control small interfering RNA (siRNA; si-FOXD3 and si-control) were synthesized by Shanghai GenePharma Co., Ltd. miR-425-5p mimics and the antisense oligonucleotide of miR-425-5p (ASO-miR-425-5p) were commercially synthesized by Shanghai GenePharma Co., Ltd. Negative control miRNA (miR-NC) containing a scramble sequence of miRNA was used as the control for miR-425-5p mimics transfection. ASO-NC containing a scramble sequence of miRNA was used as the control for ASO-miR-425-5p. The FOXD3 3' untranslated region (3'UTR) harboring the miR-425-5p target sequence and the seed sequence mutated type (FOXD3 3'UTR mut) were synthesized by Shanghai GenePharma Co., Ltd., and inserted into the pmiR-GLO vector (Promega Corporation). The primers and oligonucleotides used during the study are presented in Table II.

HCCLM3 cells at a density of $70 \%$ were transfected with miR-425-5p mimics (50 nM) or ASO-miR-425-5p (100 nM), FOXD3 expressing plasmid $(2 \mu \mathrm{g} /$ well, 12 -well plates; $4 \mu \mathrm{g} /$ well, 6 -well plates) or si-FOXD3 (100 $\mathrm{nM})$. In the rescue experiment, cells were transfected with miR-425-5p mimics (50 nM) and FOXD3 (1 $\mu \mathrm{g} /$ well, 12-well plates). For reverse transcription-quantitative PCR (RT-qPCR), RNA was isolated $48 \mathrm{~h}$ following transfection.

$R N A$ isolation and $R T-q P C R$. Total RNA was isolated using TRIzol ${ }^{\circledR}$ reagent (Invitrogen; Thermo Fisher Scientific, Inc.) according to the manufacturer's protocols. Briefly, for tissue samples, the tissue section was first cut into small pieces and ground in liquid nitrogen. Then, the samples were homogenized in TRIzol and RNA was extracted. cDNA was synthesized using the Moloney murine leukemia virus (M-MLV) reverse transcriptase (Promega Corporation). Briefly, $2 \mu \mathrm{g}$ RNA with primers was incubated at $70^{\circ} \mathrm{C}$ for $5 \mathrm{~min}$, then the M-MLV RT enzyme, M-MLV 5X Reaction Buffer, dNTPs, RNasin (Promega Corporation) and Nuclease-Free Water were added to a final volume of $25 \mu \mathrm{l}$, which was incubated for $60 \mathrm{~min}$ at $42^{\circ} \mathrm{C}$. qPCR was performed using an SYBR Premix Ex Taq kit (Takara Biotechnology Co., Ltd.). qPCR was conducted as follows: $94^{\circ} \mathrm{C}$ for $3 \mathrm{~min}$, followed by 40 cycles of $94^{\circ} \mathrm{C}$ for $30 \mathrm{sec}, 56^{\circ} \mathrm{C}$ for $30 \mathrm{sec}$, and $72^{\circ} \mathrm{C}$ for $30 \mathrm{sec}$. GAPDH and U6 were used as internal controls for mRNA and miRNA quantification, respectively. The relative expression was calculated using the $2^{-\Delta \Delta \mathrm{Cq}}$ method (40). The primers and oligonucleotides used are listed in Table II.

Western blotting. At $48 \mathrm{~h}$ following transfection, cells were lysed with RIPA buffer (Beyotime Institute of Biotechnology). The protein concentrations were measured with the Bradford assay (Bio-Rad Laboratories, Inc.). Protein samples (30 $\mu \mathrm{g})$ were separated via 10\% SDS-PAGE and then transferred onto PVDF membranes. Membranes were incubated with blocking buffer (5\% skim milk) at room temperature for $2 \mathrm{~h}$. The membranes were then incubated overnight at $4^{\circ} \mathrm{C}$ with primary antibodies against FOXD3 (1:1,000; cat. no. ab64807), 
Table I. Clinical characteristics of patients with hepatocellular carcinoma and association with miRNA-425-5p levels.

\begin{tabular}{|c|c|c|c|}
\hline Clinical parameters & miR-425-5p high $(n=19)$ & miR-425-5p low $(n=11)$ & P-value \\
\hline Age (years) & & & 0.429 \\
\hline$<50$ & 11 & 6 & \\
\hline$\geq 50$ & 8 & 5 & \\
\hline Sex & & & 0.725 \\
\hline Male & 15 & 9 & \\
\hline Female & 4 & 2 & \\
\hline TNM grade & & & $0.022^{\mathrm{a}}$ \\
\hline $\mathrm{I}+\mathrm{II}$ & 14 & 8 & \\
\hline III+IV & 5 & 3 & \\
\hline
\end{tabular}

${ }^{a} \mathrm{P}<0.05 . \mathrm{miR}$, microRNA.

Table II. Primers and oligonucleotide sequences.

\begin{tabular}{|c|c|c|}
\hline Name & & Sequences $\left(5^{\prime}-3^{\prime}\right)$ \\
\hline \multirow[t]{2}{*}{ FOXD3-plasmid } & Sense: & CGGGATCCATGACCCTCTCCGGCGGCG \\
\hline & Antisense: & CGGAATTCCTATTGCGCCGGCCATTTGGCT \\
\hline \multirow[t]{2}{*}{ FOXD3-qPCR } & Forward: & GCCCCCAACGCCTACCTTCC \\
\hline & Reverse: & АТTТСССТСССАТССССАCG \\
\hline \multirow[t]{2}{*}{ GAPDH-qPCR } & Forward: & ATGACATCAAGAAGGTGGTGAAGCAGG \\
\hline & Reverse: & GCGTCAAAGGTGGAGGAGTGGGT \\
\hline \multirow[t]{2}{*}{ si-FOXD3 } & Forward: & ACGGCCGAGGACGTGGACATC \\
\hline & Reverse: & GATGTCCACGTCCTCGGCCGT \\
\hline \multirow[t]{2}{*}{ miR-NC mimics } & Forward: & UCACAACCUCCUAGAAAGAGUAGA \\
\hline & Reverse: & UCUACUCUUUCUAGGAGGUUGUGA \\
\hline \multirow[t]{2}{*}{ miR-425-5p mimics } & Forward: & AAUGACACGAUCACUCCCGUUGA \\
\hline & Reverse: & UCUUCGGGUGUGAUCGUGUCAUU \\
\hline ASO-control & & CAGUACUUUUGUGUAGUACAA \\
\hline ASO-miR-425-5p & & AAUGACACUUUUUGCCUUUUUGA \\
\hline \multirow[t]{2}{*}{ miR-425-5p-RT } & & GTCGTATCCAGTGCAGGGTCCGAGGTGCA \\
\hline & & CTGGATACGACTCTTCGGGT \\
\hline U6-RT & & AACGCTTCACGAATTTGCGTG \\
\hline miR-425-5p-qPCR & & GCCCGCAATGACACGATCACTC \\
\hline \multirow[t]{2}{*}{ U6-qPCR } & Forward: & GCTCGCTTCGGCAGCACA \\
\hline & Reverse: & GAGGTATTCGCACCAGAGGA \\
\hline \multirow[t]{2}{*}{ FOXD3 3'UTR wt } & Forward: & UCAAAAAGGCAAAAAGUGUCAUU \\
\hline & Reverse: & AATGACACTTTTTGCCTTTTTGA \\
\hline \multirow[t]{2}{*}{ FOXD3 3'UTR mut } & Forward: & UCAAAAAGGCAAAAACACAGUAU \\
\hline & Reverse: & ATACTGTGTTTTTGCCTTTTTGA \\
\hline
\end{tabular}

qPCR, quantitative PCR; RT, reverse transcription; miR-425-5p, microRNA-425-5p; si, small interfering RNA; ASO, antisense oligonucleotide; FOXD3, forkhead box D3; 3'UTR, 3' untranslated region; wt, wild-type; mut, mutant.

E-cadherin (1:1,000; cat. no. ab15148), Vimentin $(1: 1,000$; cat. no. ab92547) and GAPDH (1:500; cat. no. ab181602), and then with goat anti-rabbit horseradish peroxidase-conjugated antibodies (1:1,000; cat. no. ab205718) at room temperature for $2 \mathrm{~h}$. All antibodies were purchased from Abcam. GAPDH was used as an internal control. Proteins were visualized by enhanced chemiluminescence (Applygen Technologies, Inc.). LabWorks 4.0 image acquisition and analysis software was used to quantify band density (UVP, LLC).

Luciferase reporter assay. HCCLM3 cells were co-transfected with miR-425-5p mimics (50 nM), ASO-miR-425-5p (100 nM) 
A

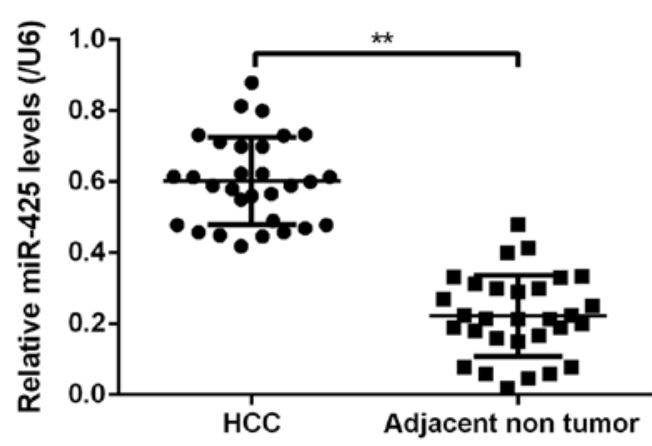

B

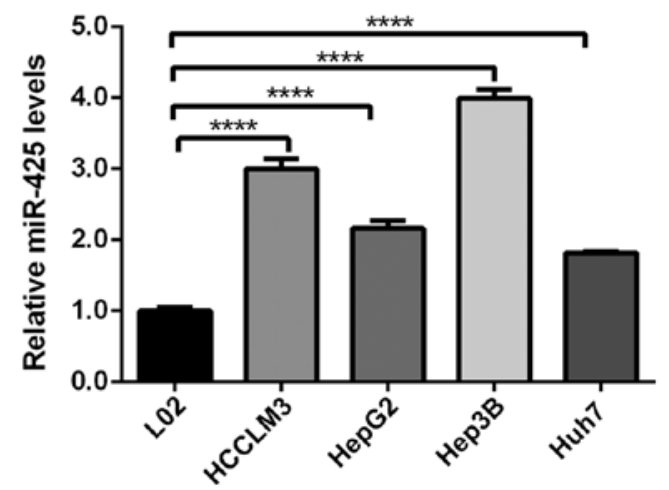

Figure 1. miR-425-5p is upregulated in HCC tissues. (A) Expression of miR-425-5p in HCC tissues and nontumor tissues (n=30/group) as determined via RT-qPCR analysis. U6 was used as an internal control. (B) Expression of miR-425-5p in human normal liver cells (L02), liver cancer cells (HepG2) and HCC cell lines (HCCLM3, Hep3B, Huh7) as determined via RT-qPCR analysis. Data are presented as the mean \pm standard error of the mean of three independent experiments. ${ }^{* *} \mathrm{P}<0.01,{ }^{* * * *} \mathrm{P}<0.0001$. HCC, hepatocellular carcinoma; miR-425-5p, microRNA-425-5p; RT-qPCR, reverse transcription-quantitative PCR.

or their respective controls, and pMIR-GLO-FOXD3 3'UTR or pMIR-GLO-FOXD3 3'UTR mut reporter (100 ng/well, 24-well plates) with Lipofectamine 2000 . The hRluc-neo was used as an internal control. Then, $48 \mathrm{~h}$ following transfection, the luciferase activities were determined using the Dual-Glo Luciferase Reporter Assay system (Promega Corporation). The results were expressed as relative luciferase activity (firefly luciferase/Renilla luciferase).

MTT assay and colony formation assay. MTT and colony formation assays were performed as previously described (41,42). Briefly, HCCLM3 cells were transfected with plasmids or oligonucleotides as aforementioned. Then, $24 \mathrm{~h}$ following transfection, the cells were plated in 96-well plates at a density of 3,000 cells/well. The viability of cells at 24, 48 and $72 \mathrm{~h}$ was evaluated using an MTT assay at $570 \mathrm{~nm}$. Briefly, $10 \mu \mathrm{l}$ MTT reagent $(10 \mathrm{mg} / \mathrm{ml})$ was added to each well prior to incubation at $37^{\circ} \mathrm{C}$ for $4 \mathrm{~h}$, following which $100 \mu 1$ dimethyl sulfoxide was added into each well. The absorbance was detected at $570 \mathrm{~nm}$ using a microplate reader (BioTek Instruments, Inc.). Cell viability was expressed as the relative $\mathrm{A}_{570}$ in experimental cells compared with in control cells [viable cells $(\%)=\left(\mathrm{abs}_{\text {sample }}-\mathrm{abs}_{\text {blank }}\right) /\left(\mathrm{abs}_{\text {control }}-\mathrm{abs}_{\text {blank }}\right)$ $\mathrm{x} 100]$. For the colony formation assay, at $24 \mathrm{~h}$ following transfection, cells were plated in 12-well plates at a density of 300 cells/well in triplicate. The culture medium was changed every 3 days. At $\sim 2$ weeks later, the cells were stained with crystal violet for $20 \mathrm{~min}$ at room temperature, and colonies containing $\geq 50$ cells were counted. The colony formation rate was expressed as colony number compared to the cells plated in each well [(colony number/cell number added x100)].

Migration and invasion assays. HCCLM3 cells were transfected with the aforementioned plasmids or oligonucleotides. Then, at $24 \mathrm{~h}$ following transfection, 5x10 ${ }^{4}$ HCCLM3 cells were seeded into the upper chamber of Transwell chamber inserts (pore size, $8 \mu \mathrm{m}$ ) without or with Matrigel (used for invasion assays). DMEM with $10 \%$ FBS was added to the upper chamber, whereas DMEM with 20\% FBS was added to the lower chamber. The chambers were maintained in $5 \% \mathrm{CO}_{2}$ at $37^{\circ} \mathrm{C}$ for $24 \mathrm{~h}$ and then stained with $0.1 \%$ crystal violet at room temperature for $20 \mathrm{~min}$. The cells on the lower side of the filter were counted under a microscope; for each sample, five fields was counted and then averaged. Images were captured under a light microscope (magnification, $\mathrm{x} 40$; Leica Microsystems GmbH).

Flow cytometry analysis. HCCLM3 cells were transfected as indicated and then seeded into 6-well plates (70\% confluence) and cultured for $48 \mathrm{~h}$. Then, the cells were collected for apoptosis analysis using an Annexin V-FITC apoptosis detection kit (BD Biosciences) according to the manufacturer's protocols. Briefly, $5 \mu \mathrm{l}$ of Annexin V-FITC was added to a $100-\mu 1$ cell solution ( $1 \times 10^{5}$ cells) and incubated at room temperature for $15 \mathrm{~min}$ in the dark. Then, $5 \mu \mathrm{l}$ PI was added to the cell solution and incubated at room temperature for $15 \mathrm{~min}$, before cells were washed with PBS. Cell apoptosis was evaluated using a BD FACSCalibur ${ }^{\mathrm{TM}}$ flow cytometer (BD Biosciences), and data were analyzed using FlowJo version 10 software (FlowJo LLC).

Statistical analysis. All data were presented as the mean \pm SEM. Statistical analyses were performed using paired Student's t-tests, $\chi^{2}$ test and one-way ANOVA with Bonferroni post hoc analysis. $\mathrm{P}<0.05$ was considered to indicate a statistically significant difference.

\section{Results}

miR-425-5p is upregulated in HCC tissues and cells. To study the expression profile of miR-425-5p in HCC, its expression levels were determined in $30 \mathrm{HCC}$ tissues and paired adjacent nontumor tissues via RT-qPCR analysis. As presented in Fig. 1A, the expression levels of miR-425-5p were significantly increased in HCC tissue compared with in adjacent nontumor tissues. The associations between miR-425-5p expression and clinical parameters were then analyzed. As presented in Table I, the expression of miR-425-5p was associated with clinical stage $(\mathrm{P}<0.01)$. Similarly, the levels of miR-425-5p were significantly upregulated in HCC and liver cancer cell lines (Huh7, Hep3B, HCCLM3 and HepG2) compared with a normal liver cell line (L02; Fig. 1B). These data indicated that miR-425-5p expression was upregulated in HCC. 
A

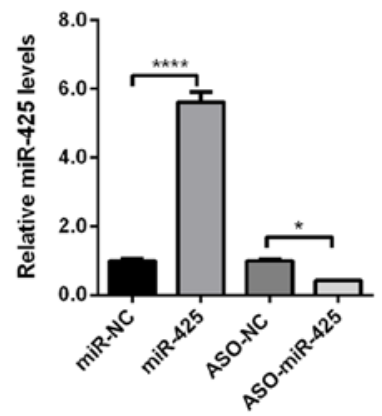

B

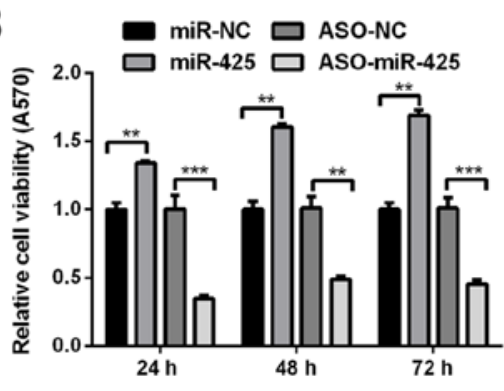

C

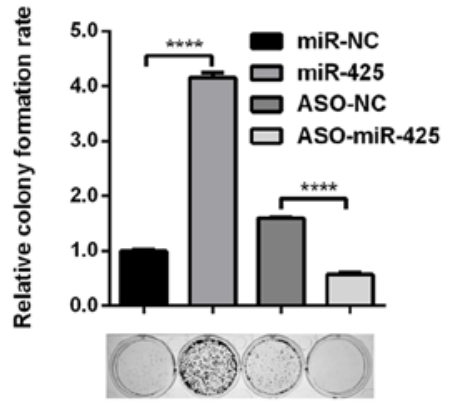

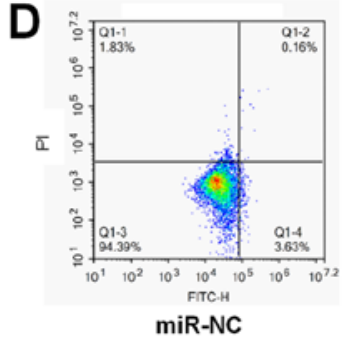

miR-NC

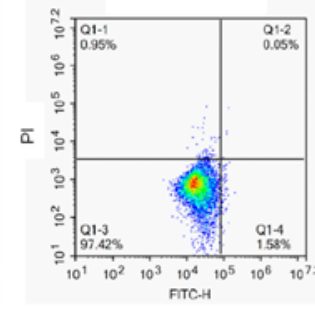

miR-425

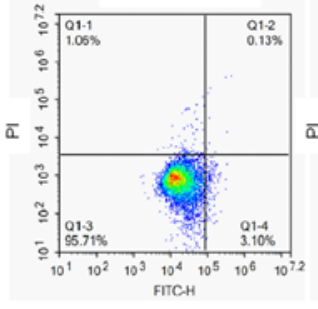

ASO-NC

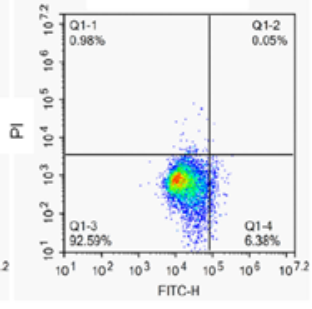

ASO-miR-425

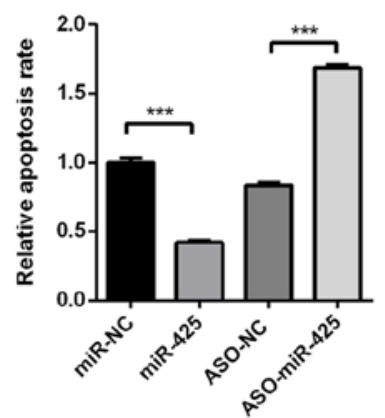

E
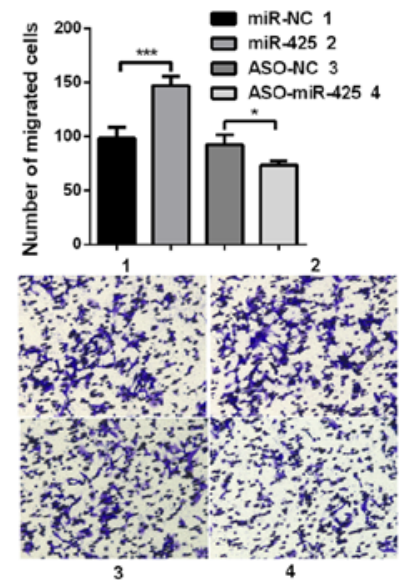

F

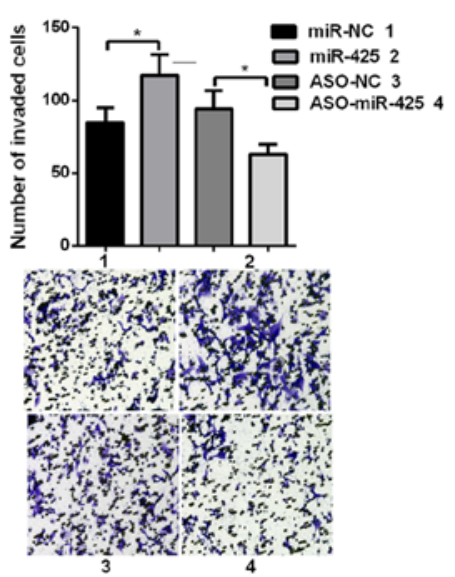

G

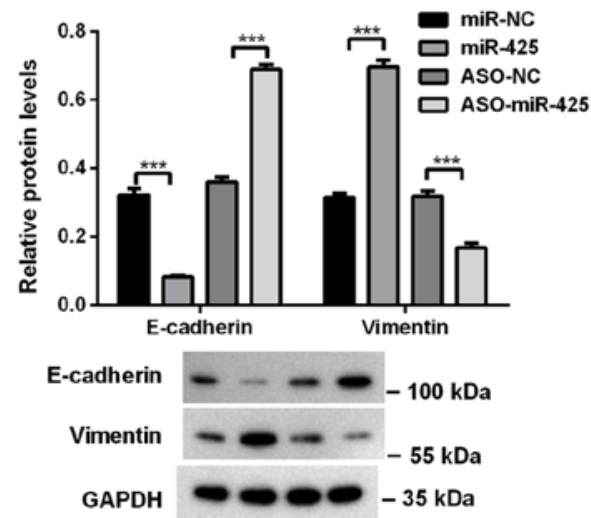

Figure 2. miR-425-5p promotes the proliferation, migration and invasion of hepatocellular carcinoma cells. (A) miR-425-5p expression in HCCLM3 cells following transfection with miR-425-5p mimics or ASO-miR-425-5p, as determined via reverse transcription-quantitative PCR analysis. (B) HCCLM3 cells were transfected with miR-425-5p mimics or ASO-miR-425-5p, and cell viability was assessed using an MTT assay. (C) Colony formation ability was evaluated using a colony formation assay. (D) HCCLM3 cells were transfected with miR-425-5p mimics or ASO-miR-425-5p and cell apoptosis was measured via flow cytometry. X-axis: Annexin V-FITC; y-axis: Propidium iodide. HCCLM3 cells were transfected with miR-425-5p mimics or ASO-miR-425-5p, and the (E) migratory and (F) invasive abilities of cells were determined using Transwell assays. Numbers in the associated key indicate the origin of the Transwell images. (G) HCCLM3 cells were transfected with miR-425-5p mimics or ASO-miR-425-5p, and the protein levels of E-cadherin and Vimentin were analyzed via western blotting. Data are presented as the mean \pm standard error of the mean of three independent experiments. ${ }^{*} \mathrm{P}<0.05,{ }^{* *} \mathrm{P}<0.01,{ }^{* * * *} \mathrm{P}<0.001,{ }^{* * * * *} \mathrm{P}<0.0001$. miR-425-5p, microRNA-425-5p; ASO, antisense oligonucleotide; NC, negative control; miR-NC, miR-425-5p mimics NC.

miR-425-5p promotes cell proliferation, migration and invasion in vitro. To investigate the roles of $\mathrm{miR}-425-5 \mathrm{p}$ in HCC progression, its effects on the malignant behaviors of HCC cells were determined. miR-425-5p mimics or ASO-miR-425-5p were transfected into HCCLM3 cells. RT-qPCR analysis revealed that the expression of miR-425-5p was significantly increased following transfection with miR-425-5p mimics compared with miR-NC, but was significantly decreased following ASO-miR-425-5p transfection compared with ASO-NC (Fig. 2A). MTT and colony formation assays revealed that the viability and colony formation ability of HCCLM3 cells were significantly increased when miR-425-5p was overexpressed, whereas miR-425-5p knockdown significantly decreased viability and colony formation (Fig. 2B and C). Additionally, to address whether the increase in cell proliferation was associated with cell apoptosis, flow cytometry analysis was used to evaluate the apoptosis of HCCLM3 cells. Annexin V staining revealed that the number of apoptotic cells was significantly decreased when HCCLM3 cells were transfected with miR-425-5p mimics, whereas transfection of ASO-miR-425-5p induced opposing effects (Fig. 2D). Furthermore, Transwell assays revealed that the migratory and invasive abilities of HCCLM3 cells were promoted following miR-425-5p overexpression, but were decreased by miR-425-5p knockdown (Fig. 2E and F). To determine the mechanisms underlying the effects of miR-425-5p on the migration and invasion of cells, the expression of epithelial-mesenchymal transition markers were evaluated via western blot analysis. As presented in Fig. 2G, overexpression of miR-425-5p significantly increased Vimentin expression but decreased E-cadherin expression, whereas miR-425-5p knockdown increased E-cadherin expression, but 
A

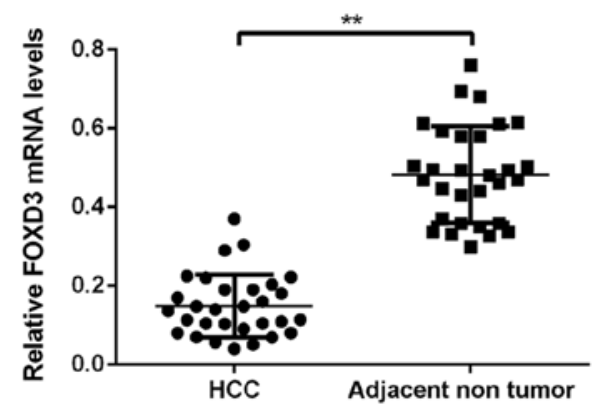

B FOXD3 3'UTR wt UCAAAAAGgCAAAAAGUGUCAUU

miR-425 AGUUGCCCUCACUAGCACAGUAA

FOXD3 3'UTR mut UCAAAAAGGCAAAAACACAGUAU

conservation among vertebrates

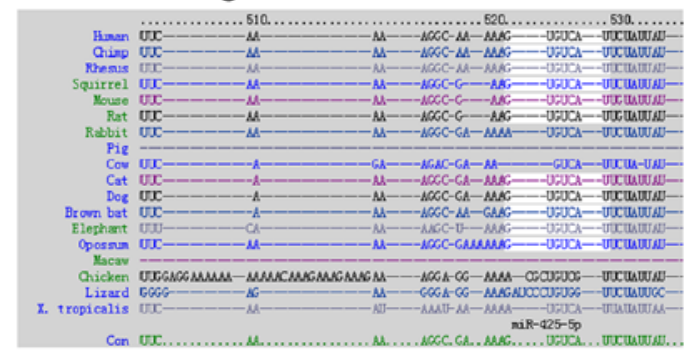

C

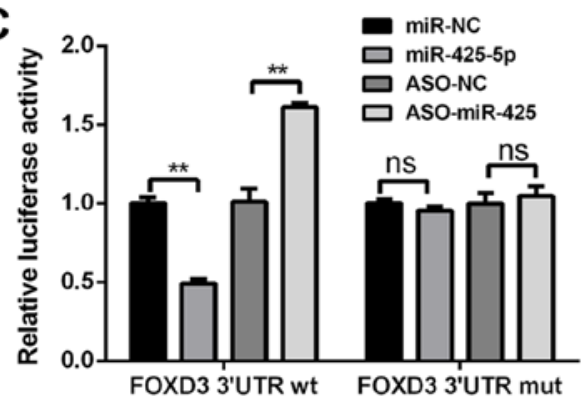

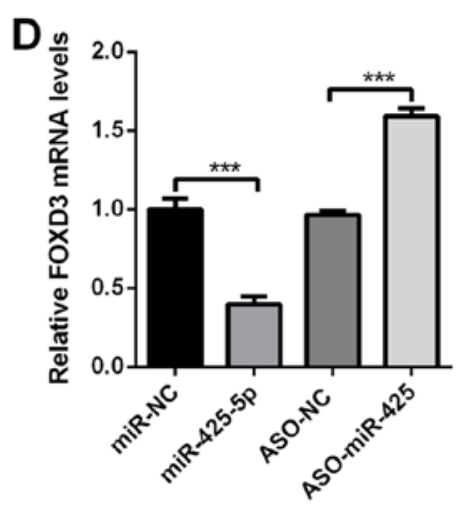

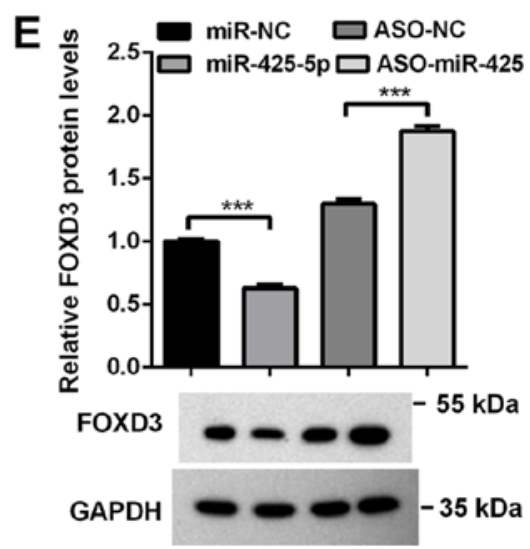

Figure 3. FOXD3 is a direct target of miR-425-5p. (A) mRNA levels of FOXD3 in HCC tissues and matched tumor-adjacent tissues (n=30/group) as determined via RT-qPCR analysis. (B) Predicted binding site of miR-425-5p in the 3'UTR of FOXD3 mRNA and the mut sequence. (C) HCCLM3 cells were co-transfected with the indicated reporter plasmids, and miR-425-5p mimic or ASO-miR-425-5p, and the luciferase activity was determined via dual-luciferase assays. HCCLM3 cells were transfected with miR-425-5p mimic or ASO-miR-425-5p, and (D) mRNA and (E) protein levels of FOXD3 were determined via RT-qPCR and western blot analyses, respectively. Data are presented as the mean \pm standard error of the mean of three independent experiments. ${ }^{* *} \mathrm{P}<0.01$, ${ }^{* * *} \mathrm{P}<0.001$; ns, not significant. miR-425-5p, microRNA-425-5p; ASO, antisense oligonucleotide; NC, negative control; miR-NC, miR-425-5p mimics NC; FOXD3, forkhead box D3; HCC, hepatocellular carcinoma; RT-qPCR, reverse transcription-quantitative PCR; ns, no significance; wt, wild-type; mut, mutant; 3'UTR, 3' untranslated region.

decreased Vimentin expression. These data demonstrated that miR-425-5p promoted the proliferation, migration and invasion of HCC cells.

FOXD3 is a target of miR-425-5p in HCC cells. To investigate the underlying mechanisms by which miR-425-5p promotes HCC development, the potential targets of miR-425-5p were predicted using the TargetScan and PicTar databases. Among the predicted targets, FOXD3 was selected, as it has been reported as a tumor suppressor gene in various tumors, including HCC $(29,30)$. Its expression in HCC tissues was determined via RT-qPCR analysis; as presented in Fig. 3A, its expression was significantly decreased in HCC tissues compared with the adjacent nontumor tissues. Bioinformatics analysis revealed that there was one putative binding site in the 3'UTR of FOXD3 mRNA (Fig. 3B); this site is conserved among vertebrates. First, a dual luciferase assay was conducted to determine whether miR-425-5p directly targets FOXD3. As presented in Fig. 3C, overexpression of miR-425-5p significantly decreased the luciferase activity of FOXD3 3'UTR (by 60\%), whereas blocking miR-425-5p markedly increased the luciferase activities of FOXD3 3'UTR (by $\sim 50 \%$ ). Conversely, when the potential binding sites for miR-425-5p in the FOXD3 3'UTR were mutated, miR-425-5p did not significantly affect the luciferase activities of FOXD3 3'UTRmut (Fig. 3C). Then, the effects of miR-425-5p on endogenous FOXD3 mRNA and protein were investigated via RT-qPCR and western blot analyses. RT-qPCR revealed that the mRNA levels of FOXD3 were significantly decreased when miR-425-5p was overexpressed, whereas knockdown of miR-425-5p resulting in opposing effects (Fig. 3D). Western blot analysis also revealed that overexpression of miR-425-5p significantly decreased, whereas knockdown of miR-425-5p increased the protein levels of FOXD3 (Fig. 3E). It was also revealed that its promoter was hypermethylated in HCC tissues and HCCLM3 cells (Table SII). These results indicated that miR-425-5p inhibits FOXD3 expression partly by binding directly to the FOXD3 3'UTR.

FOXD3 suppresses HCC cell proliferation, migration and invasion in vitro. To determine whether FOXD3 was involved in the effects of miR-425-5p on HCC cells, a FOXD3 overexpression plasmid (pcDNA3.0/FOXD3) and si-FOX3D were constructed, and the efficiency of these vectors was demonstrated by RT-qPCR and western blot analyses (Fig. 4A). The effects of FOXD3 on the malignant behaviors of HCC cells were determined. MTT and colony formation assays revealed that overexpression of FOXD3 in HCCLM3 cells significantly 

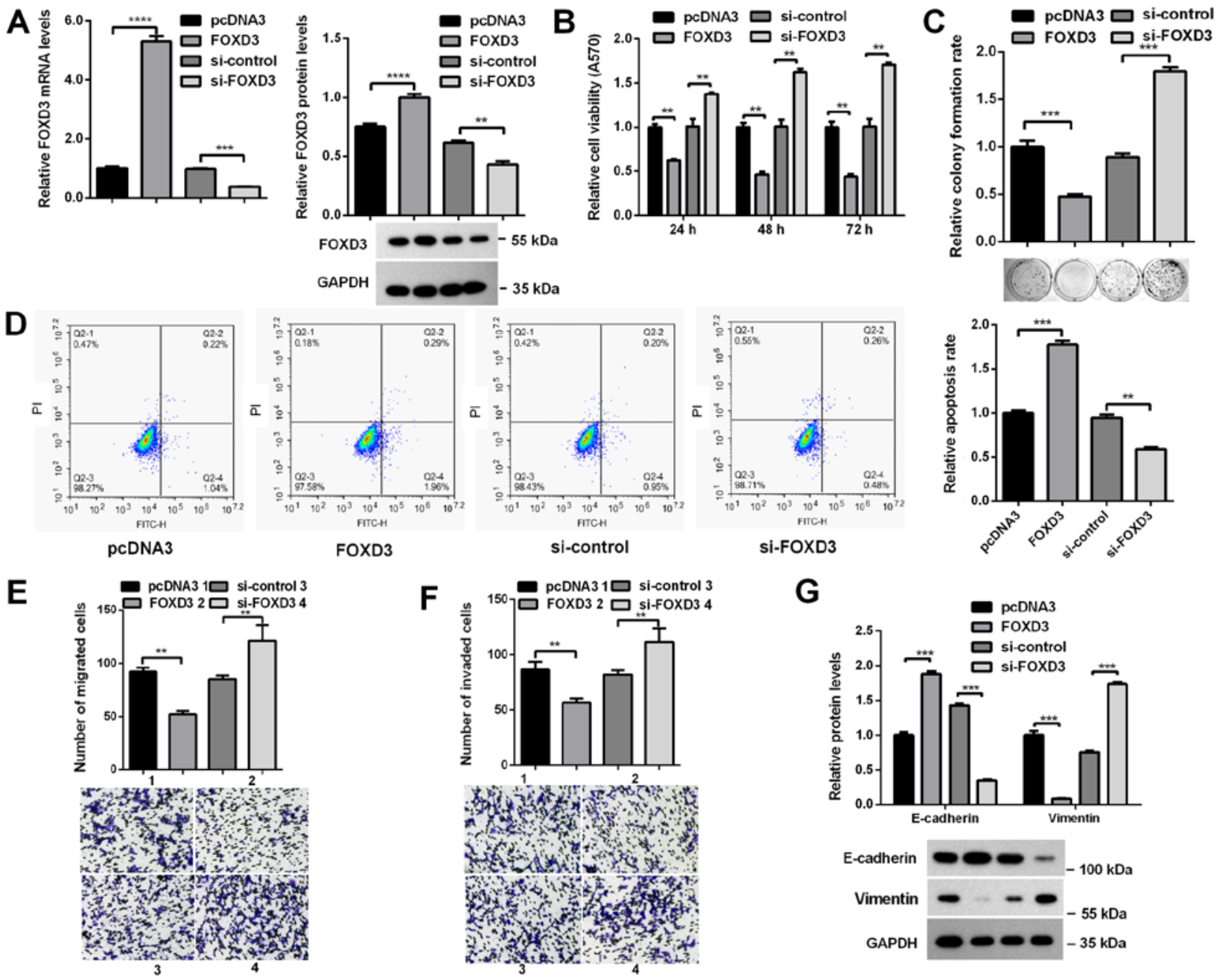

Figure 4. FOXD3 suppresses the proliferation, migration and invasion of hepatocellular carcinoma cells. (A) HCCLM3 cells were transfected with pcDNA3.0/FOXD3 or si-FOXD3, and $48 \mathrm{~h}$ later, FOXD3 protein levels were evaluated via western blotting. GAPDH was used as a control. (B) HCCLM3 cells were transfected and the cell viability was determined by MTT assay at 24, 48 and $72 \mathrm{~h}$ post-transfection. (C) HCCLM3 cells were transfected and the cell colony formation ability was determined using a colony formation assay. (D) HCCLM3 cells were transfected and the apoptosis rate was determined via flow cytometry. HCCLM3 cells were transfected and the (E) migratory and (F) invasive abilities of cells were evaluated using Transwell assays. (G) HCCLM3 cells were transfected and the protein levels of E-cadherin and Vimentin were analyzed via western blotting. Data are presented as the mean \pm standard error of the mean of three independent experiments. ${ }^{* *} \mathrm{P}<0.01,{ }^{* * *} \mathrm{P}<0.001,{ }^{* * * *} \mathrm{P}<0.0001$. FOXD3, forkhead box D3; si, small interfering RNA.

decreased the viability and colony formation ability of cells, whereas knockdown of FOXD3 induced opposing effects (Fig. 4B and C). Flow cytometry analysis demonstrated that the apoptosis rate of cells was significantly increased when FOXD3 was overexpressed, but decreased following FOXD3 knockdown (Fig. 4D). Furthermore, the migratory and invasive abilities of HCCLM3 cells were significantly decreased following overexpression of FOXD3, whereas FOXD3 knockdown significantly increased the migratory and invasive abilities of HCCLM3 cells (Fig. 4E and F). Western blot analysis revealed that overexpression of FOXD3 significantly increased E-cadherin, but decreased Vimentin expression, whereas FOXD3 knockdown downregulated E-cadherin and upregulated Vimentin expression (Fig. 4G). These data indicated that FOXD3 inhibited the proliferation, migration and invasion of HCC cells.

miR-425-5p promotes HCC cell proliferation, migration and invasion via the downregulation of FOXD3 expression. miR-425-5p represses endogenous FOXD3 mRNA expression; however, the overexpression of FOXD3 without its 3'UTR can recover the protein levels of FOXD3. Therefore, to investigate whether the effects of miR-425-5p on the progression of HCC were mediated by downregulating FOXD3, HCCLM3 cells were co-transfected with miR-425-5p and pcDNA3.0/FOXD3, which encodes the FOXD3 coding sequence but lacks the 3'UTR. Western blot analysis revealed that transfection with FOXD3 significantly reversed the effects of miR-425-5p on FOXD3 expression (Fig. 5A). MTT and colony formation assays revealed that the restoration of FOXD3 expression significantly rescued the effects of miR-425-5p on cell viability and colony formation ability (Fig. 5B and C). Flow cytometry analysis demonstrated that the inhibition of cell apoptosis by miR-425-5p was attenuated by FOXD3 overexpression (Fig. 5D). Transwell assays revealed that the positive effects of miR-425-5p on cell migration and invasion were suppressed by FOXD3 overexpression (Fig. 5E and F). Similar results were observed for the expression of E-cadherin and Vimentin (Fig. 5G). Collectively, these results indicated that miR-425-5p promoted the proliferation, migration and invasion of HCC cells by repressing FOXD3 expression. 

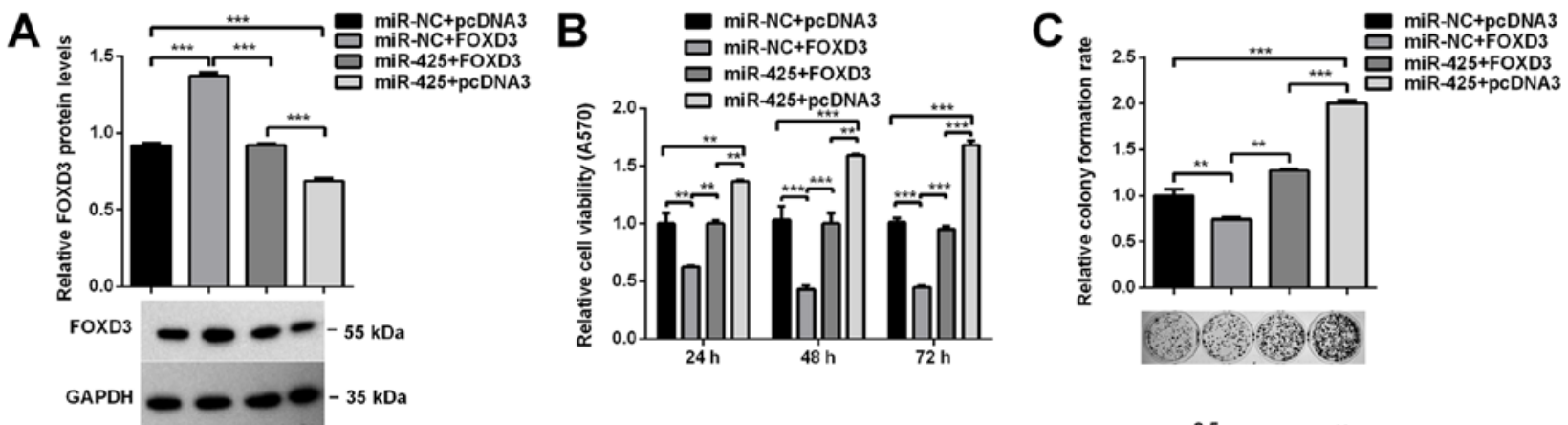

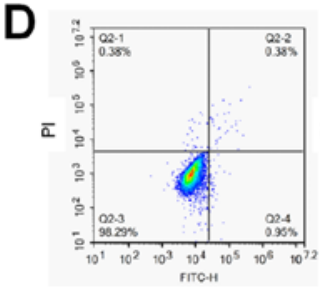

miR-NC+pcDNA3

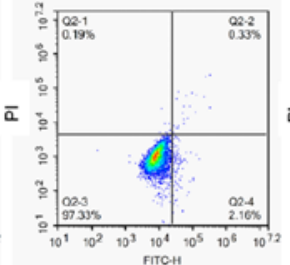

miR-NC+FOXD3

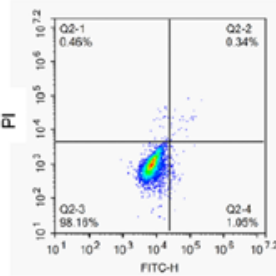

miR-425+FOXD3

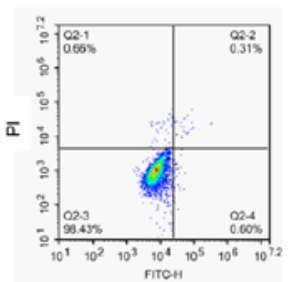

miR-425+pcDNA3

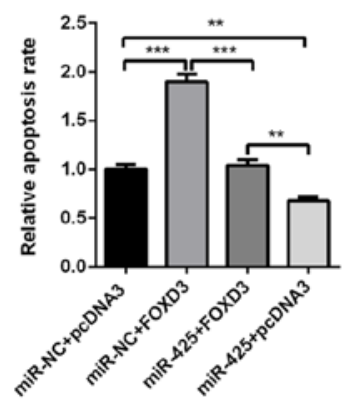

E

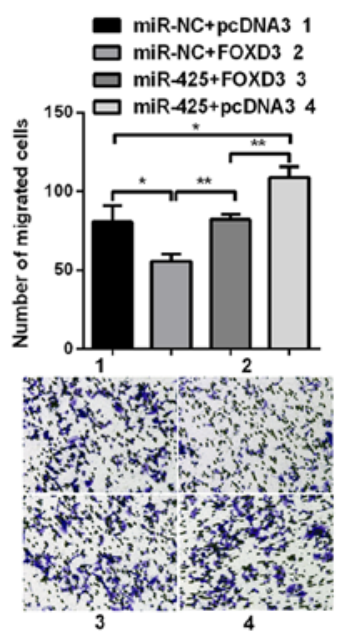

$\mathbf{F}$

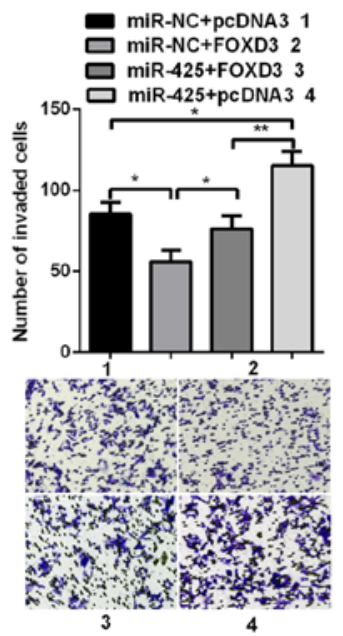

G

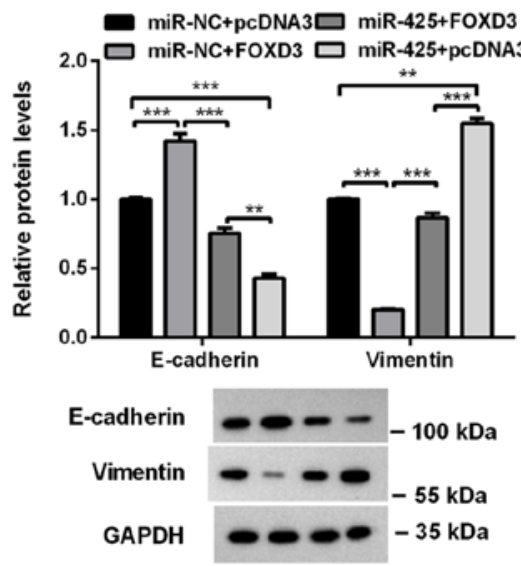

Figure 5. miR-425-5p promotes HCC cell proliferation, migration and invasion via the downregulation of FOXD3. (A) HCCLM3 cells were transfected and FOXD3 protein levels were evaluated via western blotting. (B) Cell viability was analyzed using MTT assays. (C) Cell colony formation ability was determined using a colony formation assay. (D) Cell apoptosis was evaluated via flow cytometry. (E) Migration and (F) invasion of HCC cells as determined using Transwell assays. (G) Protein levels of E-cadherin and Vimentin as determined via western blotting. Data are presented as the mean \pm standard error of the mean of three independent experiments. ${ }^{*} \mathrm{P}<0.05,{ }^{* *} \mathrm{P}<0.01,{ }^{* * *} \mathrm{P}<0.001$. miR, microRNA; NC, negative control; FOXD3, forkhead box D3; HCC, hepatocellular carcinoma.

\section{Discussion}

It has been reported that miR-425-5p expression is dysregulated in various cancers (19-26); its expression was increased in the majority of cases, with the exception of nasopharyngeal carcinoma and melanoma. In the present study, it was observed that miR-425-5p was upregulated in HCC, consistent with a previous study (21); however, the specific mechanisms responsible for its upregulation in HCC require further exploration. Furthermore, miR-425-5p was reported to be associated with the pathogenesis and progression of numerous cancers $(19-21,24,26)$; however, its role varies in different cancers. For example, miR-425-5p promoted cell proliferation, migration and invasion by targeting PTEN and the lysine deubiquitinase CYLD in gastric cancer $(19,20)$. miR-425-5p promoted the invasion and metastasis of tumor cells via the suppressor of cancer cell invasion protein (SCAI)-mediated dysregulation of multiple signaling pathways in HCC (21), whereas it acted as a tumor suppressor in nasopharyngeal carcinoma and melanoma cells by targeting hepatoma-derived growth factor and insulin-like growth factor 1 , respectively $(24,26)$. In the present study, it was reported that miR-425-5p promoted HCC cell proliferation, migration and invasion by targeting transcription factor FOXD3, suggesting that it serves an oncogenic role.

miRNAs are post-transcriptional regulators that function by degrading target mRNAs or inhibiting the translation of target mRNAs. To search for targets of miR-425-5p, TargetScan and PicTar, two online software tools, were employed, which identified FOXD3 as a potential target. First, its expression was decreased in HCC tissues compared with adjacent nontumor tissues, consistent with a previous study that reported that the downregulation of FOXD3 in HCC due to promoter hypermethylation (30). It was further revealed that the promoter of FOXD3 was hypermethylated in HCC tissues and HCCLM3 cells. Whether there are other mechanisms responsible for its downregulation in HCC requires further study. Then, luciferase 
assays, and RT-qPCR and western blot analyses revealed that miR-425-5p bound directly to and suppressed the expression of endogenous FOXD3 mRNA. Additionally, rescue experiments indicated that the promoting effects of miR-425-5p on HCC progression in HCCLM3 cells were mediated via the downregulation of FOXD3. As the miR-425-5p levels are different in different HCC cells, the degree to which malignant behaviors are rescued by FOXD3 may vary. With previous findings that miR-425-5p promotes the invasion and metastasis of HCC cells by targeting SCAI and regulating its downstream signaling pathways (21), miR-425-5p may promote HCC development via more than one target; however, whether the other targets are involved in the effects of miR-425-5p on HCC cells requires further investigation. Of note, in the present study, it was demonstrated that miR-425-5p increased the viability and colony formation ability of HCCLM3 cells; flow cytometry analysis suggested that this may be partly due to the inhibition on apoptosis. Conversely, in a previous study, the proliferation and colony number of SMMC-7721 and HCCLM3 cells were not affected by miR-425-5p (21). This difference may be a result of different cell culture conditions, but the specific reason requires further investigation. In addition, when the effects of miR-425-5p on the colony formation ability of HCCLM3 cells were investigated, it was observed that ASO-NC markedly increased colony formation compared with miR-NC. The reasons for this are unclear, as the two oligonucleotides are scrambles of the experimental molecules and share no homology to other host genes; however, it may be that the molecules induced distinct cytotoxic effects on cells, as ASO-NC is single-stranded, whereas miR-NC is double-stranded.

It was reported that FOXD3 has an important role in the development of numerous cancers (29-37). In colon cancer, FOXD3 suppressed the invasive ability and inhibited the apoptosis of cells by inhibiting the epidermal growth factor receptor-Ras-Raf-mitogen activated protein kinase kinase (MEK)-ERK signaling pathway, or regulating miR-214 expression and function $(32,33)$. FOXD3 can also suppress cell invasion by inhibiting the expression of the short isoform 2 of doublecortin-like kinase 1 , which is a cancer stem cell marker (34). In melanoma, FOXD3 was shown to be involved in cancer cell proliferation and migration via the regulation of Twist-related protein 1, C-X-C chemokine receptor 4 and paired box gene 3; its expression was controlled by MEK activity (38-40). In HCC, FOXD3 was reported to suppress cell proliferation, invasion and metastasis by regulating miR-137 expression (29). The present findings revealed similar effects on the malignant behaviors of HCC cells; however, whether miR-425-5p functions via an miR-425-5p/FOXD3/miR-137 axis or via other pathways requires further investigation.

In conclusion, the present results demonstrated that miR-425-5p was downregulated in HCC, and that miR-425 suppressed HCC cell proliferation, migration and invasion by targeting FOXD3. This study may improve understanding of the molecular mechanisms underlying HCC development, providing novel insight for the treatment of HCC.

\section{Acknowledgements}

Not applicable.

\section{Funding}

This work was supported by the Scientific and Technological Project of Henan Province (grant no. 172102310098).

\section{Availability of data and materials}

All data generated or analyzed during this study are included in this published article.

\section{Authors' contributions}

HW, JS, WZ and JL performed the experiments. HW, HN and $\mathrm{NC}$ analyzed and interpreted the data. HW and JS drafted the manuscript. All authors read and approved the final manuscript.

\section{Ethics approval and consent to participate}

Written informed consent was obtained from each patient. The study was approved by the Ethics Committee of Henan Provincial People's Hospital.

\section{Patient consent for approval}

Not applicable.

\section{Competing interests}

The authors declare that they have no competing interests.

\section{References}

1. Vasuri F, Visani M, Acquaviva G, Brand T, Fiorentino M, Pession A, Tallini G, D'Errico A and de Biase D: Role of microRNAs in the main molecular pathways of hepatocellular carcinoma. World J Gastroenterol 24: 2647-2660, 2018.

2. Croce CM: Causes and consequences of microRNA dysregulation in cancer. Nat Rev Genet 10: 704-714, 2009.

3. Giovannini C, Fornari F, Dallo R, Gagliardi M, Nipoti E, Vasuri F, Coadă CA, Ravaioli M, Bolondi L and Gramantieri L: MiR-199-3p replacement affects E-cadherin expression through Notch1 targeting in hepatocellular carcinoma. Acta Histochem 120: 95-102, 2018.

4. Guan J, Liu Z, Xiao M, Hao F, Wang C, Chen Y, Lu Y and Liang J: MicroRNA-199a-3p inhibits tumorigenesis of hepatocellular carcinoma cells by targeting ZHX1/PUMA signal. Am J Transl Res 9: 2457-2465, 2017.

5. Ghosh A, Dasgupta D, Ghosh A, Roychoudhury S, Kumar D, Gorain M, Butti R, Datta S, Agarwal S, Gupta S, et al: MiRNA199a-3p suppresses tumor growth, migration, invasion and angiogenesis in hepatocellular carcinoma by targeting VEGFA, VEGFR1, VEGFR2, HGF and MMP2. Cell Death Dis 8: e2706, 2017.

6. Ren K, Li T, Zhang W, Ren J, Li Z and Wu G: miR-199a-3p inhibits cell proliferation and induces apoptosis by targeting YAP1, suppressing Jagged1-Notch signaling in human hepatocellular carcinoma. J Biomed Sci 23: 79, 2016.

7. Kim JH, Badawi M, Park JK, Jiang J, Mo X, Roberts LR and Schmittgen TD: Anti-invasion and anti-migration effects of miR-199a-3p in hepatocellular carcinoma are due in part to targeting CD151. Int J Oncol 49: 2037-2045, 2016.

8. Henry JC, Park JK, Jiang J, Kim JH, Nagorney DM, Roberts LR, Banerjee S and Schmittgen TD: miR-199a-3p targets CD44 and reduces proliferation of CD44 positive hepatocellular carcinoma cell lines. Biochem Biophys Res Commun 403: 120-125, 2010.

9. Fornari F, Milazzo M, Chieco P, Negrini M, Calin GA, Grazi GL, Pollutri D, Croce CM, Bolondi L and Gramantieri L: MiR-199a-3p regulates mTOR and c-Met to influence the doxorubicin sensitivity of human hepatocarcinoma cells. Cancer Res 70: 5184-5193, 2010. 
10. Callegari E, D'Abundo L, Guerriero P, Simioni C, Elamin BK, Russo M, Cani A, Bassi C, Zagatti B, Giacomelli L, et al: miR-199a-3p modulates MTOR and PAK4 pathways and inhibits tumor growth in a hepatocellular carcinoma transgenic mouse model. Mol Ther Nucleic Acids 11: 485-493, 2018.

11. Varshney A, Panda JJ, Singh AK, Yadav N, Bihari C, Biswas S, Sarin SK and Chauhan VS: Targeted delivery of microRNA-199a-3p using self-assembled dipeptide nanoparticles efficiently reduces hepatocellular carcinoma in mice. Hepatology 67: 1392-1407, 2018.

12. Luo C, Yin D, Zhan H, Borjigin U, Li C, Zhou Z, Hu Z, Wang P, Sun Q, Fan J, et al: microRNA-501-3p suppresses metastasis and progression of hepatocellular carcinoma through targeting LIN7A. Cell Death Dis 9: 535, 2018.

13. Liu H, Chen W, Zhi X, Chen EJ, Wei T, Zhang J, Shen J, Hu LQ, Zhao B, Feng XH, et al: Tumor-derived exosomes promote tumor self-seeding in hepatocellular carcinoma by transferring miRNA-25-5p to enhance cell motility. Oncogene 37: 4964-4978, 2018.

14. Lin XJ, Fang JH, Yang XJ, Zhang C, Yuan Y, Zheng L and Zhuang SM: Hepatocellular carcinoma cell-secreted exosomal MicroRNA-210 promotes angiogenesis in vitro and in vivo. Mol Ther Nucleic Acids 11: 243-252, 2018.

15. Fang JH, Zhang ZJ, Shang LR, Luo YW, Lin YF, Yuan Y and Zhuang SM: Hepatoma cell-secreted exosomal microRNA-103 increases vascular permeability and promotes metastasis by targeting junction proteins. Hepatology 68: 1459-1475, 2018.

16. Fang T, Lv H, Lv G, Li T, Wang C, Han Q, Yu L, Su B, Guo L, Huang S, et al: Tumor-derived exosomal miR-1247-3p induces cancer-associated fibroblast activation to foster lung metastasis of liver cancer. Nat Commun 9: 191, 2018.

17. Bartel DP: MicroRNAs: Genomics, biogenesis, mechanism, and function. Cell 116: 281-297, 2004.

18. Lee YS and Dutta A: MicroRNAs in cancer. Annu Rev Pathol 4 199-227, 2009

19. Yan YF, Gong FM, Wang BS and Zheng W: MiR-425-5p promotes tumor progression via modulation of CYLD in gastric cancer. Eur Rev Med Pharmacol Sci 21: 2130-2136, 2017.

20. Zhang Z, Li Y, Fan L, Zhao Q, Tan B, Li Z and Zang A: microRNA-425-5p is upregulated in human gastric cancer and contributes to invasion and metastasis in vitro and in vivo. Exp Ther Med 9: 1617-1622, 2015.

21. Fang F, Song T, Zhang T, Cui Y, Zhang G and Xiong Q: MiR-425-5p promotes invasion and metastasis of hepatocellular carcinoma cells through SCAI-mediated dysregulation of multiple signaling pathways. Oncotarget 8: 31745-31757, 2017.

22. Sun L, Jiang R, Li J, Wang B, Ma C, Lv Y and $\mathrm{Mu} \mathrm{N}$ : MicoRNA-425-5p is a potential prognostic biomarker for cervical cancer. Ann Clin Biochem 54: 127-133, 2017.

23. Liu L, Zhao Z, Zhou W, Fan X, Zhan Q and Song Y: Enhanced expression of miR-425 promotes esophageal squamous cell carcinoma tumorigenesis by targeting SMAD2. J Genet Genomics 42: 601-611, 2015

24. Zhu W, Ma Y, Zhuang X and Jin X: MicroRNA-425 is downregulated in nasopharyngeal carcinoma and regulates tumor cell viability and invasion by targeting hepatoma-derived growth factor. Oncol Lett 15: 6345-6351, 2018.

25. Quan J, Li Y, Pan X, Lai Y, He T, Lin C, Zhou L, Zhao L, Sun S, Ding Y, et al: Oncogenic miR-425-5p is associated with cellular migration, proliferation and apoptosis in renal cell carcinoma. Oncol Lett 16: 2175-2184, 2018.
26. Liu P, Hu Y, Ma L, Du M, Xia L and Hu Z: miR-425 inhibits melanoma metastasis through repression of PI3K-Akt pathway by targeting IGF-1. Biomed Pharmacother 75: 51-57, 2015.

27. Weigel D and Jäckle H: The fork head domain: A novel DNA binding motif of eukaryotic transcription factors? Cell 63: 455-456, 1990.

28. Yong JS, Intriago-Baldeón DP and Lam EW: FOXD3 controls pluripotency through modulating enhancer activity. Stem Cell Investig 3: 17, 2016.

29. Liu LL, Lu SX, Li M, Li LZ, Fu J, Hu W, Yang YZ, Luo RZ Zhang CZ and Yun JP: FoxD3-regulated microRNA-137 suppresses tumour growth and metastasis in human hepatocellular carcinoma by targeting AKT2. Oncotarget 5: 5113-5124, 2014.

30. He G, Hu S, Zhang D, Wu P, Zhu X, Xin S, Lu G, Ding Y and Liang L: Hypermethylation of FOXD3 suppresses cell proliferation, invasion and metastasis in hepatocellular carcinoma. Exp Mol Pathol 99: 374-382, 2015.

31. Yin H, Meng T, Zhou L, Zhao F, Li X, Li Y, Hu M, Chen H and Song D: FOXD3 regulates anaplastic thyroid cancer progression. Oncotarget 8: 33644-33651, 2017.

32. He GY, Hu JL, Zhou L, Zhu XH, Xin SN, Zhang D, Lu GF, Liao WT, Ding YQ and Liang L: The FOXD3/miR-214/MED19 axis suppresses tumour growth and metastasis in human colorectal cancer. Br J Cancer 115: 1367-1378, 2016.

33. Li K, Guo Q, Yang J, Chen H, Hu K, Zhao J, Zheng S, Pang X, Zhou S, Dang Y and Li L: FOXD3 is a tumor suppressor of colon cancer by inhibiting EGFR-Ras-Raf-MEK-ERK signal pathway. Oncotarget 8: 5048-5056, 2017.

34. Sarkar S, O'Connell MR, Okugawa Y, Lee BS, Toiyama Y, Kusunoki M, Daboval RD, Goel A and Singh P: FOXD3 regulates CSC marker, DCLK1-S, and invasive potential: Prognostic implications in colon cancer. Mol Cancer Res 15: 1678-1691, 2017.

35. Kubic JD, Little EC, Kaiser RS, Young KP and Lang D: FOXD3 promotes PAX3 expression in melanoma cells. J Cell Biochem 117: 533-541, 2016.

36. Kubic JD, Lui JW, Little EC, Ludvik AE, Konda S, Salgia R, Aplin AE and Lang D: PAX3 and FOXD3 Promote CXCR4 Expression in Melanoma. J Biol Chem 290: 21901-21914, 2015.

37. Weiss MB, Abel EV, Dadpey N and Aplin AE: FOXD3 modulates migration through direct transcriptional repression of TWIST1 in melanoma. Mol Cancer Res 12: 1314-1323, 2014.

38. Agarwal V, Bell GW, Nam JW and Bartel DP: Predicting effective microRNA target sites in mammalian mRNAs. Elife 4: e05005, 2015.

39. Krek A, Grün D, Poy MN, Wolf R, Rosenberg L, Epstein EJ, MacMenamin P, da Piedade I, Gunsalus KC, Stoffel M and Rajewsky N: Combinatorial microRNA target predictions. Nat Genet 37: 495-500, 2005.

40. Livak KJ and Schmittgen TD: Analysis of relative gene expression data using real-time quantitative PCR and the 2(-Delta Delta C(T)) method. Methods 25: 402-408, 2001

41. Li H, Yang T, Shang D and Sun Z: miR-1254 promotes lung cancer cell proliferation by targeting SFRP1. Biomed Pharmacother 92: 913-918, 2017.

42. Chen H, Yang Y, Wang J, Shen D, Zhao J and Yu Q: miR-130b-5p promotes proliferation, migration and invasion of gastric cancer cells via targeting RASAL1. Oncol Lett 15: 6361-6367, 2018. 